\title{
Pregnancy: a therapeutic dilemma
}

\author{
Ligia Brzezińska-Wcisłoํㅜ, Martyna Zbiciak-Nylec², Dominika Wcisło-Dziadecka², Natalia Salwowska ${ }^{1}$
}

${ }^{1}$ Chair and Department of Dermatology, School of Medicine, Medical University of Silesia, Katowice, Poland

${ }^{2}$ Andrzej Mielęcki Silesian Independent Public Hospital, Katowice, Poland

${ }^{3}$ Department of Skin Structural Studies, Chair of Cosmetology, School of Pharmacy with Division of Laboratory Medicine in Sosnowiec, Medical University of Silesia in Katowice, Poland

Adv Dermatol Allergol 2017; XXXIV (5): 433-438

DOI: https://doi.org/10.5114/ada.2017.71108

\begin{abstract}
Treatment during pregnancy is problematic. The Food and Drug Administration established drug categories to help in the treatment process. First-generation antihistamines are considered safe but they have sedative properties. Second-generation antihistamines cause less adverse reactions but besides cetirizine and loratadine they belong to category C. All retinoids should be avoided during pregnancy due to the risk of fetal malformations. Antimalarial drugs should be considered based on the clinical data. Sulfones can be considered as safe for use during pregnancy only with proper monitoring. Prednisone is administered in pregnancy. Other glucocorticosteroids have a different safety profile. Cyclosporine A treatment should be reserved as rescue therapy in severe stages of the disease. Treatment during pregnancy should be precise when it comes to pregnant woman and safe for the fetus.
\end{abstract}

Key words: pregnancy, antihistamine, drug categories, retinoids, antimalarial drugs.

\section{Introduction}

Treatment during pregnancy is challenging. Almost every medical intervention while pregnant or breast feeding can cause adverse reactions to the child. The Food and Drug Administration (FDA) established drugs categories $A, B, C, D, X$ and $N$ reflecting the risk of fetal injury. Class A consists of drugs that have failed to demonstrate a risk to the fetus in the first trimester of pregnancy in adequate and well-controlled human studies. In class B, animal studies have failed to demonstrate a risk or have shown an adverse effect but failed in well-controlled studies in pregnant women. Class C drugs have shown in animal studies an adverse effect on the fetus and there are no adequate and well-controlled studies in humans. In class D there is positive evidence of the human fetal risk but benefits may warrant use of the drug in pregnant women. Class $X$ contains drugs that are contraindicated in pregnancy. There is a large group of drugs in class $\mathrm{N}$ which contains dermatological drugs that are not yet classified. Dermatologists are often confronted with the need of treatment of pregnant women. The therapeutic options are deferral of therapy for conditions that do not require immediate therapy, maintain current therapy if considered safe, verification and replacement of therapy, and also sustained breastfeeding and returning after therapy. The aim of this article is to discuss the safety of dermatological drugs in the light of current knowledge.

\section{Antihistamines}

Antihistamines are used to treat itching. Itch is one of the common symptoms during pregnancy and treatment is very challenging when it comes to diagnosing and treating. Antihistamines are also used in allergy therapy and for treatment of persistent vomiting during pregnancy [1]. First-generation antihistamines are considered as safe during pregnancy $[2,3]$. They include diphenhydramine, cyproheptadine, promethazine, chlorpheniramine, tripelennamine and hydroxyzine. The FDA categorizes first-generation antihistamine drugs based on the risk of fetal defects (Table 1) [1, 4]. Drugs such as diphenhydramine and chlorpheniramine are class B $[5,6]$. Promethazine and hydroxyzine belong to class $C$ drugs due to lack of well-controlled studies on humans [1]. First-generation antihistamines are well known of their sedative properties as a result of their applicability to cross the blood-brain barrier. In one of the larger prospective studies of 50 pregnant woman taking diphenhydramine and chlorpheniramine, transient irritability and drowsiness was observed. No serious side effects to newborn were shown [7]. In another study which evalu-

Address for correspondence: Prof. Ligia Brzezińska-Wcisło MD, PhD, Department of Dermatology, School of Medicine, Medical University of Silesia, 20-24 Francuska St, 40-027 Katowice, Poland, e-mail: dermatologia@spskm.katowice.pl

Received: 4.10.2016, accepted: 30.10 .2017$. 
Table 1. First-generation antihistamines categories according to FDA

\begin{tabular}{lc}
\hline First-generation antihistamines & FDA categories \\
\hline Chlorpheniramine & $\mathrm{B}$ \\
\hline Cyproheptadine & $\mathrm{B}$ \\
\hline Diphenhydramine & $\mathrm{B}$ \\
\hline Hydroxyzine & $\mathrm{C}$ \\
\hline Promethazine & $\mathrm{C}$ \\
\hline Tripelennamine & $\mathrm{B}$ \\
\hline
\end{tabular}

ated breastfeeding mothers who were taking antihistamines due to seasonal allergy, the results were similar. In $22.6 \%$ of patients, increased irritability and drowsiness was reported [8]. There is also a theoretical risk of impairing milk production in women who were on firstgeneration antihistamine therapy due to influence on dopamine secretion into the brain. This risk is theoretical only because several studies show that antihistamines do not influence lactation [9]. Scientists from Michigan studied the exposure to chlorpheniramine (class B) in the first trimester. No correlation to higher incidence of fetus defects was found. There are no data on the safety of first-generation antihistamines during breastfeeding. Due to multitude of studies proving safety of the drug during pregnancy, chlorpheniramine should be preferred in the first trimester [10]. There are reports that diphenhydramine (class B) is associated with a higher incidence of cleft palate, but the data are not equivocal and are anecdotal in later studies. Therapeutic doses do not correspond with a higher concentration in breast milk, while manufacturers warn against its use in women while breast-feeding. When it comes to phenothiazine (class C), its use should be avoided during pregnancy. Hydroxyzine, in the first trimester, increases the risk of fetal defects. It can also cause hypotension, myoclonus, hypoxia, and central nervous system (CNS) depression. In animal studies, hydroxyzine was teratogenic. There are no data on the drug concentration in breast milk. Loratadine, cetirizine, fexofenadine, levocetirizine and desloratadine are second-generation antihistamines (Table 2). The risk of their applicability to cross the blood-brain barrier is smaller, and thus have minimal effects on CNS. Only 3\% of cetirizine, $0.1 \%$ of fexofenadine and $1.1 \%$ of loratadine

Table 2. Second-generation antihistamines categories according to FDA

\begin{tabular}{lc}
\hline Second-generation antihistamines & FDA categories \\
\hline Cetirizine & $\mathrm{B}$ \\
\hline Fexofenadine & $\mathrm{C}$ \\
\hline Loratadine & $\mathrm{B}$ \\
\hline Levocetirizine & $\mathrm{B}$ \\
\hline Desloratadine & $\mathrm{C}$ \\
\hline
\end{tabular}

makes its way to the brain. Therapeutic doses are too low to cause adverse effects. Nevertheless, while taking large doses during pregnancy, excessive irritability, tremors and drowsiness should be considered as a reason for discontinuation of therapy [9]. Table 2 shows secondgeneration antihistamines as categorized by the FDA. Fexofenadine and desloratadine are classified as class $C$ drugs. In children of mothers using fexofenadine, reduced birth weight and increased mortality was observed [1]. Cetirizine and loratadine are the only non-sedative antihistamines with B category. There were reports of an increased risk of hypospadias in children of mothers using loratadine, but further studies did not confirm this $[1,11-13]$. For years loratadine and cetirizine have been preferred as first-line drugs used during the second and third trimester [11]. Caution must be taken and high doses of antihistamines should not be administered before delivery. Those drugs can cause an oxytocin effect, stimulating uterine contractions what can lead to premature birth. A relation between the use of antihistamines 2 weeks before the birth and the retrolental fibroplasia in preterm infants was observed [2]. Moreover there is a risk of infant withdrawal syndrome manifested with annoyance, nervousness, and problems with feeding. First generation of antihistamines should be avoided in breastfeeding women because of the potential sedative properties [5, 6]. Loratadine, fexofenadine and cetirizine are preferred drugs during breastfeeding.

\section{Retinoids}

Acne is a very common disorder in dermatological practice. Antibiotics and retionids are most commonly used drugs in acne vulgaris. Isotretinoin is considered class $X$ in the FDA categories. This drug should be avoided during pregnancy and women who are planning motherhood. An increased risk of miscarriage in the first trimester results from the significant fetal malformations [5]. According to the European Directive and the recommendations of the Office for Registration of Medicinal Products, each patient in the childbearing age range is obliged to use effective contraception for at least a 1 month before starting on isotretionoin treatment, throughout its duration, as well as, 1 month after end of treatment. Two pregnancy tests during treatment and one 5 weeks after the end of treatment to exclude pregnancy are necessary to be performed by the dermatologist $[14,15]$. We should provide continuous education about the dangers connected with becoming pregnant during treatment [11]. Since 1988 acitretin has been available in almost every European country and primarily used in psoriasis treatment. Like all systemic retinoids, acitretin also has a teratogenic effect. In women of childbearing age, absolute pregnancy prevention one month before, after, and during the treatment, is required. It is also noted that those women should be deterred from 
acitretin therapy due to 2 years' contraception period $[11,16]$. Retinoids are also contraindicated during breastfeeding. Acitretion should be particularly avoided due to potential cumulative toxicity, which can lead to renal failure, hepatotoxicity (vitamin A overdose), increased intracranial pressure, osteoarticular pain and increased susceptibility to respiratory tract infections [9]. No birth defects were reported concerning paternal retinoid use. Still contraception is recommended throughout use of retinoid therapy.

\section{Antimalarial drugs}

For women with systemic lupus erythematosus, antimalarial drugs are first-line therapy. Treatment discontinuation is accompanied by the risk of exacerbation and most experts recommend continuing the therapy even if pregnancy occurs during treatment [17]. In the case of disease remission, women who are pregnant or planning motherhood should be treated with corticosteroids on a shorter-term basis. In that case, steroids are safer due to numerous available data on the safety of steroids during pregnancy [5]. According to FDA, antimalarial drugs are class $C$ of the FDA categories. Hydroxychloroquine does not increase the risk of specific congenital fetal malformations. It must be noted that during hydroxychloroquine treatment we should monitor changes in retina. In a large prospective study in female patients with systemic lupus erythematosus (SLE) treated with hydroxychloroquine, reduction of prednisolone was possible [18]. Another positive effect of antimalarial treatment is a lower risk of heart block in newborns with neonatal lupus erythematosus (NLE) [19]. Published studies support physicians in their decision on safe hydroxychloroquine therapy continuation in pregnant women $[11,19]$. Usage of antimalarial drugs during lactation is controversial. Based on the current literature, antimalarial drugs have a minimal impact on the newborn. Only $0.005 \%$ to $0.35 \%$ of hydroxychloroquine passes into breast milk. A theoretical total daily dose for a child is $0.16 \mathrm{mg} / \mathrm{kg} /$ day (2.9\%). As a reference point, the pediatric total weekly dose of antimalarial drug (hydroxychloroquine) for children is $5 \mathrm{mg} / \mathrm{kg} /$ week. Concerns about the use of antimalarial drugs during lactation are related with their slow elimination, meaning, there is a theoretical risk of toxicity for newborns. In 1988, the World Health Organization (WHO) made a statement that women treated with antimalarial drugs should avoid breastfeeding. But the American Academy of Pediatrics (AAP) guidelines from 1992 consider antimalarial drugs as safe during lactation [9, 20].

\section{Sulfones}

Dapsone belongs to a class of drugs known as sulfones. The drug is first-line therapy for Duhring's disease. It is an antibiotic and an anti-inflammatory drug which makes it ideal for use in treatment of many chronic inflammatory diseases, autoimmune disorders, particularly vesicular diseases. With proper monitoring sulfones can be considered as safe for use during pregnancy [21, 22]. According to the FDA, dapsone is a class C drug. Animal studies with high dapsone doses have not shown teratogenicity of this drug, but there is lack of studies in humans [11]. Limited data on use of sulfones in pregnant women do not allow unambiguous determination of the safety of sulfones. Previously, it has been described that there is a low incidence of adverse reactions in newborns of mothers treated with dapsone but hemolytic disease of the newborn, neonatal jaundice and methemoglobinemia should be mentioned. However, those cases are isolated [21, 23]. Dapsone passes through the placenta and into breast milk at 15\% [24]. This drug has a long half-life (20 h) which is associated with an increased risk of accumulation [21]. Therefore, the WHO determined dapsone as dangerous during lactation [20]. The AAP indicates that this drug should be avoided in children from the hyperbilirubinemia/glucose-6-phosphate dehydrogenase deficiency risk group $[25,26]$. Kardos et al. analyzed current data on the treatment of pemphigus vugaris in pregnancy and the impact of therapy on a newborn. They compared the treatment regimen in 49 pregnant women with pemphigus. In 37 (75\%) out of 49 patients, prednisolone was used in therapy. In the rest of the patients, combined treatment of plasmapheresis and sulfones was chosen. No side effects to the infants was reported [24].

\section{Glucocorticoids}

Systemic corticosteroids in moderate doses are considered safe in pregnant women with persistent itching or autoimmune disease. But individual drugs are in different FDA categories e.g. prednisone (class B), prednisolone (class B), dexamethasone (class C), betamethasone (class C). In a large study conducted in Michigan, a clear connection between birth defects and corticosteroids treatment in pregnant women in the first trimester of pregnancy, was noted. In a large study conducted in Michigan, a clear connection between birth defects and corticosteroids treatment in pregnant women in the first trimester of pregnancy, was noted: in women treated with prednisone $(n=236)$, prednisolone $(n=143)$ and methylprednisolone $(n=222)$ [27]. In 2000, meta-analysis showed that the treatment with corticosteroids has a three-fold increased risk of cleft in the craniofacial region if treatment took place 4 weeks before pregnancy and up to 12 weeks after birth. Labial fusion and adhesion in the fetus takes place between $5^{\text {th }}$ and $7^{\text {th }}$ week of pregnancy, while palate formation occurs during weeks 8-12. The absolute risk of this complication is low and around 1 for 1000 live births. However, the recent Hviid and Molgaard-Nielsoen publication does not show the relation between use of steroids and cleft lip/palate [28]. According to many au- 
thors, prednisone is preferred during pregnancy because its penetration through the placenta is limited by enzymes, while dexamethasone almost completely passes through. Children of mothers treated with corticosteroids during pregnancy should be additionally monitored for adrenal insufficiency due to iatrogenic hormone suppression. Prednisone may also cause premature birth, premature amniotic rupture, intrauterine growth restriction (IUGR), as well as diabetes, hypertension, pre-eclampsia and eclampsia. Many clinicians recommend reducing the dose to $7.5 \mathrm{mg} /$ day in long-term treatment and avoiding doses higher than $20 \mathrm{mg} /$ day [29]. Prednisone is metabolized into prednisolone. Studies have shown that a small amount of the metabolite is transferred to breast milk [30]. For women treated with doses of 10-80 mg of prednisone, relative blood levels in children was $0.02 \%$ to $0.074 \%$ (0.002-0.059 mg), which is less than $10 \%$ of infantile endogenous cortisol levels [31]. Previous studies have shown no adverse reactions in breast-fed infants, however mothers were treated with low doses of corticosteroids (5-10 mg/day) [32, 33]. In order to minimize exposure and avoid peak serum concentration occurring within the $1^{\text {st }} \mathrm{h}$ after drug ingestion, it is recommended that the mother should wait $4 \mathrm{~h}$ before breastfeeding. During that time, drug concentration in the milk falls to the level in maternal plasma [34]. The pediatrician society believe that corticosteroids are safe for breastfeeding women [2].

\section{Immunosuppressants}

\section{Cyclosporine A}

According to the FDA, cyclosporine A (CSA) is a class C drug. Most data concerning CsA includes transplant recipients in whom generally higher doses (8-10 mg/kg/ daz) are used as compared to dermatological patients. Treatment with cyclosporine A during pregnancy carries the risk of premature birth and low birth weight of the newborn $[35,36]$. In the light of previous studies, this drug does not cause fetal defects. A large group of children of mothers who were treated with cyclosporine $A$ during pregnancy were observed in early development stages. Neurocognitive, behavioral, immunological, or nephrological disorders were not detected [37-39]. Cyclosporine $A$ is considered as a drug of choice in case of oral treatment necessity in severe psoriasis in pregnant women. However we should remember that CSA can induce hypertension in mothers and this treatment should be reserved as rescue therapy in severe stages of disease. This drug is excreted into breast milk and should not be prescribed during lactation. Data show that CSA occurs in breast milk in different concentration. In seven newborns, which had been exposed to the drug during breastfeeding, nephrotoxicity was not recorded, which was assessed by testing creatinin levels from the first week until the completion of breastfeeding. Furthermore, no damage to the thymus was found although this has been reported in animal studies [40]. Although there are cases of properly developing children, the AAP and other experts treat CSA as cytotoxic drugs and recommend avoiding their use during lactation [25, 32]. In cases where the mother decides to breastfeed the child she must be monitored for signs of CSA toxicity including edema, tremor, hirsutism and hypertension. Plasma CSA levels should be also controlled [34].

\section{Azathioprine}

Azathioprine is reported as a class D drug. It passes through the placenta in its inactive form. Available data mainly includes women who are post transplantation or have inflammatory bowel disease and it shows that azathoprine during pregnancy may increase the risk of prematurity and low birth weight. In a small number of studies concerning azathioprine treatment in breastfeeding women, the drug concentration in breast milk is very low and does not threaten the infants, but caution is advised [41-44].

\section{Cyclophosphamide}

This drug is teratogenic when used in the first trimester. According to the FDA, cyclophosphamide is classified as a class D medication. It can cause intrauterine growth restriction and intrauterine death. Cyclophosphamide should be avoided during pregnancy, and if use occurs, treatment should be discontinued. During breastfeeding, cyclophosphamide treatment is not recommended.

\section{Methotrexate}

During methotrexate (MTX) treatment, contraception is required throughout treatment and for 3 months after its completion. The drug has is a class X medication according to the FDA. Methrotrexate therapy during pregnancy may result in spontaneous miscarriage and methotrexate embryopathy is characterized by intrauterine growth restriction, microcephaly, cranial bone hypoplasia, craniosynostosis (skull bone atresia), broad root of the nose, exophthalmus, low-set ears, jaw hypoplasia, presents of epicanthic fold, shortening of the limbs, syndactyly, and Fallot's tetralogy. Most of mothers of children with defects mentioned above were treated with MTX of at least $10 \mathrm{mg}$ per week between the $6^{\text {th }}$ and $10^{\text {th }}$ week of pregnancy. The TX treatment is contraindicated during breastfeeding [45].

\section{Antibacterial, antiviral and antifungal treatment}

Penicillins, cephalosporins, macrolides and clindamycin are considered as safe during pregnancy. Metronidazole can be safely administered only in the second and third trimester. All above drugs are class B medications according to the FDA. During breastfeeding, penicillin and cephalosporins are preferred due to small amounts 
of secretion through breast milk. Due to a great number of reports documenting safety of aciclovir, it is considered as the drug of choice during pregnancy. This drug is classified as class B. Oral antifungal drugs such as ketoconazole, fluconazole, itraconazole, nystatin and natamycin are classified as class $\mathrm{C}$ according to the FDA. In literature there are some reports of probable relation between oral nystatin use in the $8^{\text {th }}-14^{\text {th }}$ week of pregnancy and an increased risk of hypospadias in the fetus. Individual studies have shown a teratogenic effect of fluconazole in human in case of a dose higher than 400 mg/day in first trimester. While a single dose of $150 \mathrm{mg}$ given only once, such as in the case of a genital tract infection, did not show a risk for the fetus. According to available research, itraconazole in pregnant women does not increase the risk of fetal malformation but statistically often leads to miscarriage in the early stages of pregnancy. Therefore, in case of itraconazole treatment, effective contraception, is recommended. Amphotericin B is a class B drug and available data have shown no teratogenic impact on humans. It is considered as the safest antifungal drug for systemic use. There are no data concerning terbinafine use in pregnant women, but animal studies have shown no teratogenic effects of this drug [46, 47].

\section{Conflict of interest}

The authors declare no conflict of interest.

\section{References}

1. Kar S, Krishnan A, Preetha K, Mohankar A. A review of antihistamines used during pregnancy. J Pharmacol Pharmacother 2012; 3: 105-8.

2. Hale EK, Pomeranz MK. Dermatologic agents during pregnancy and lactation: an update and clinical review. Int J Dermatol 2002; 41: 197-203.

3. Lis-Swiety A, Brzezinska-Wcislo L. The safety of the antihistamines in dermatoses of pregnancy. Wiad Lek 2006; 59: 89-91.

4. Meadows M. Pregnancy and the drug dilemma. FDA Consum 2001; 35: 16-20.

5. Tyler KH, Zirwas MJ. Pregnancy and dermatologic therapy reply letter. J Am Acad Dermatol 2014; 70: 1136-7.

6. Leachman SA, Reed BR. The use of dermatologic drugs in pregnancy and lactation. Dermatol Clin 2006; 24: 167-97.

7. Ito S, Blajchman A, Stephenson M, et al. Prospective followup of adverse reactions in breast-fed infants exposed to maternal medication. Am J Obstet Gynecol 1993; 168: 1393-9.

8. Moretti ME, Liau-Chu M, Taddio A, et al. Adverse events in brest-fed infants exposed to antihistamines in maternal milk. Reprod Toxicol 1995; 9: 588.

9. Butler DC, Heller MM, Murase JE. Safety of dermatologic medications in pregnancy and lactation: Part II. Lactation. J Am Acad Dermatol 2014; 70: 417e1-10.

10. Briggs GG, Freeman RK, Yaffe SJ. Drugs in Pregnancy and Lactation. Lippincott Williams \& Wilkins, Baltimore 1998.

11. Murase JE, Heller MM, Butler DC. Safety of dermatologic medications in pregnancy and lactation: Part I. Pregnancy. J Am Acad Dermatol 2014; 70: 401.e1-14.
12. Diav-Citrin O, Shechtman S, Aharonovich A, et al. Pregnancy outcome after gestational exposure to loratadine or antihistamines: a prospective controlled cohort study. J Allergy Clin Immunol 2003; 111: 1239-43.

13. Centers for Disease Control and Prevention (CDC). Evaluation of an association between loratadine and hypospadias - United States, 1997-2001. MMWR Morb Mortal Wkly Rep 2004; 53: 219-21.

14. Kunynetz RA. A review of systemic retinoid therapy for acne and related conditions. Skin Therapy Lett 2004; 9: 1-4.

15. Brelsford M, Beute TC. Preventing and managing the side effects of isotretinoin. Semin Cutan Med Surg 2008; 27: 197-206.

16. Raguideau F, Mezzarobba M, Zureik M, et al. Compliance with pregnancy prevention plan recommendations in 8672 French women of childbearing potential exposed to acitretin. Pharmacoepidemiol Drug Saf 2015; 24: 526-33.

17. Cunningham FG, Leveno KJ, Bloom SL, Hauth JC, Gilstrap LC, Wenstrom KD (eds). Williams obstetrics. McGrawHill, New York 2005.

18. Clowse ME, Magder L, Witter F, Petri M. Hydroxychloroquine in lupus pregnancy. Arthritis Rheum 2006; 54: 3640-7.

19. Izmirly PM, Costedoat-Chalumeau N, Pisoni CN, et al. Maternal use of hydroxychloroquine is associated with a reduced risk of recurrent anti-SSA/Ro-antibody-associated cardiac manifestations of neonatal lupus. Circulation 2012; 126: 76-82.

20. Bennett P, Matheson I, Notarianni LJ, Kane A, Reinhardt D. Monographson individual drugs. In: Drugs and Human Lactation. Bennett P (eds). Elsevier, New York 1988; 219-20.

21. Wcisło-Dziadecka D, Zbiciak M, Brzezińska-Wcisło L. Zastosowanie sulfonów w wybranych jednostkach dermatologicznych - przegląd piśmiennictwa. Dermatol Klin 2014; 16: 35-40.

22. Wozel VE. Innovative use of dapsone. Dermatol Clin 2010; 28: 599-610.

23. Sanders SW, Zone JJ, Foltz RL, et al. Hemolytic anemia induced by dapsone transmitted through breast milk. Ann Intern Med 1982; 96: 465-6.

24. Kardos M, Levine D, Gürcan HM, Ahmed RA. Pemphigus vulgaris in pregnancy: analysis of current data on the management and outcomes. Obstet Gynecol Surv 2009; 64: 739-49.

25. American Academy of Pediatrics Committee on Drugs. Transfer of drugs and other chemicals into human milk. Pediatrics 2001; 108: 776-89.

26. Kaye D, Nshemerirwe R, Mutyaba T, Ndeezi G. A randomized clinical trial comparing safety, clinical and parasitological response to artemether-lumefantrine and chlorproguanil-dapsone in treatment of uncomplicated malaria in pregnancy in Mulago hospital, Uganda. J Infect Dev Ctries 2008; 2: 135-9.

27. Sasseville D. Dermatological therapy during pregnancy and lactation. In: Skin Changes and Disease in Pregnancy. Harahap M, Wallach RC (eds). Marcel Dekker, New York 1996; 249-319.

28. Hviid A, Molgaard-Nielsoen D. Corticosteroid use during pregnancy and the risk of orofacial clefts. CMAJ 2011; 183: 796-804.

29. Park-Wyllie L, Mazzotta P, Pastuszak A, et al. Birth defects after maternal exposure to corticosteroids: prospective cohort study and meta-analysis of epidemiological studies. Teratology 2000; 62: 385-92.

30. Sagraves R, Kaiser D, Sharpe GL. Prednisone and prednisolone concentrations in milk of a lactating mother. Drug Intell Clin Pharm 1981; 15: 484. 
31. Elliott $A B$, Chakravarty EF. Immunosuppressive medications during pregnancy and lactation in women with autoimmune diseases. Womens Health (Lond) 2010; 6: 431-40.

32. Moretti ME, Sgro M, Johnson DW, et al. Cyclosporine excretion into breast milk. Transplantation 2003; 75: 2144-6.

33. Munoz-Flores-Thiagarajan KD, Easterling T, Davis C, Bond EF. Breast-feeding by a cyclosporine-treated mother. Obstet Gynecol 2001; 97: 816-8.

34. Hale TW, Hartmann PE (eds.). Hale \& Hartmann's Textbook of Human Lactation. Hale Publishing, Amarillo, USA 2007.

35. Armenti VT, Radomski JS, Moritz MJ, et al. Report from the National Transplantation Pregnancy Registry (NTPR): outcomes of pregnancy after transplantation. Clin Transpl 2005: 69-83.

36. Bar OB, Hackman R, Einarson T, Koren G. Pregnancy outcome after cyclosporine therapy during pregnancy: a metaanalysis. Transplantation 2001; 71: 1051-5.

37. Nulman I, Sgro M, Barrera M, et al. Long-term neurodevelopment of children exposed in uteroto ciclosporin after maternal renal transplant. Paediatr-Drugs 2010; 12: 113-22.

38. Cochat P, Decramer S, Robert-Gnansia E, et al. Renal outcome of children exposed to cyclosporine in utero. Transplant Proc 2004; 36: 208-210.

39. Butler DC, Gupta R, Murase JE. Review of recommendations for dermatologic therapy in pregnancy. J Am Acad Dermatol 2014; 70: 1135-6.

40. Nyberg G, Haljamae U, Frisenette-Fich C, et al. Breastfeeding during treatment with cyclosporine. Transplantation 1998; 65: 253-5.

41. Peyrin-Biroulet L, Oussalah A, Roblin X, Sparrow MP. The use of azathioprine in Crohn's disease during pregnancy and in the post-operative setting: a worldwide survey of experts. Aliment Pharmacol Ther 2011; 33: 707-13.

42. Cleary BJ, Källén B. Early pregnancy azathioprine use and pregnancy outcomes. Birth Defects Res A Clin Mol Teratol 2009; 85: 647-54.

43. Christensen LA, Dahlerup JF, Nielsen MJ, et al. Azathioprine treatment during lactation. Aliment Pharmacol Ther 2008; 28: 1209-13.

44. Sau A, Clarke S, Bass J, et al. Azathioprine and breastfeeding: is it safe? BJOG 2007; 114: 498-501.

45. Hyoun SC, Običan SG, Scialli AR. Teratogen update: methotrexate. Birth Defects Res A Clin Mol Teratol 2012; 94: 187207.

46. Pilmis B, Jullien V, Sobel J, et al. Antifungal drugs during pregnancy: an updated review. J Antimicrob Chemother 2015; 70 14-22.

47. Czeizel AE, Kazy Z, Puhó E. A population-based case-control teratological study of oral nystatin treatment during pregnancy. Scand J Infect Dis 2003; 35: 830-5. 